\title{
BMJ Open Impact of approaches in improving male partner involvement in the prevention of mother-to-child transmission (PMTCT) of HIV on the uptake of PMTCT services in sub-Saharan Africa: a protocol of a systematic review and meta-analysis
}

Noah F Takah,, ${ }^{1,2}$ Iain T R Kennedy, ${ }^{3}$ Cathy Johnman ${ }^{2,3}$

To cite: Takah NF, Kennedy ITR, Johnman C. Impact of approaches in improving male partner involvement in the prevention of mother-to-child transmission (PMTCT) of HIV on the uptake of PMTCT services in sub-Saharan Africa: a protocol of a systematic review and meta-analysis. BMJ Open 2016:6:e012224.

doi:10.1136/bmjopen-2016012224

- Prepublication history for this paper is available online. To view these files please visit the journal online (http://dx.doi.org/10.1136/ bmjopen-2016-012224).

Received 25 April 2016 Revised 13 June 2016 Accepted 15 June 2016

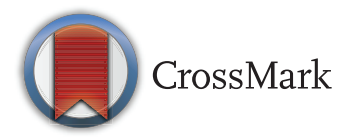

For numbered affiliations see end of article.

Correspondence to Dr Noah F Takah; takahnoah@yahoo.com

\section{ABSTRACT}

Introduction: Several studies have reported approaches used in improving the delivery of prevention of mother-to-child transmission (PMTCT) of HIV services through the involvement of male partners, but evidence from a systematic review is limited. We aim at determining the impact of male partner involvement on PMTCT in sub-Saharan Africa.

Methods and analysis: This will be a systematic review of published literature. Interventional and observational studies on male involvement in PMTCT carried out in sub-Saharan Africa will be included irrespective of the year and language of publication. OVID Medline, Embase, PschINFO, and Cochrane database of controlled trials will be searched. After manual searching of articles, authors shall be contacted for further information. 2 authors (NFT and CJ) will independently screen potential articles for eligibility using defined inclusion and exclusion criteria. Critical Appraisal Skills Programme (CASP) tools, Jadad scale and the STROBE checklist will be used for critical appraisal, and the 2 authors will independently assess the quality of articles. Authors will independently extract data from studies using a pre-established data collection form, and any discrepancies will be sorted by a third author (TRK). Outcomes will be analysed using STATA V.12.0. The random effect model will be used to produce forest plots. The heterogeneity $\chi^{2}$ statistics and $\mathrm{I}^{2}$ will be used to assess for heterogeneity. Publication bias will be assessed using funnel plots. This protocol is reported according to the Preferred Reporting Items for Systematic Reviews and Meta-Analysis Protocols (PRISMA-P) 2015 guidelines.

Ethics and dissemination: No ethical approval since included studies will be published studies that had already obtained ethical approvals. The findings will guide HIV programmes on the best approaches towards involving male partners in PMTCT with a view to improving PMTCT services in sub-Saharan Africa.

PROSPERO registration number: 42016032673.

\section{INTRODUCTION}

Rationale

Globally, there are 35 million people living with HIV, with the majority (71\%) located in sub-Saharan Africa (SSA). ${ }^{12}$ Of the 3.2 million children living with HIV globally, $91 \%$ live in SSA and in 2013, 240000 new HIV infections occurred in children which is equivalent to 1 child being infected every 2 min. ${ }^{1}$ The risk of mother-to-child transmission is between $25 \%$ and $35 \%$ in developing countries, and evidence suggests that with prevention interventions, such as antiretrovirals, the risk can be reduced to $<10 \%{ }^{3}$ Interventions directed towards the improvement of prevention of mother-to-child transmission (PMTCT) of HIV services, such as antenatal testing for pregnant women, safe delivery practices, safe infant breastfeeding practices, maternal antiretroviral therapy (ART), and infant antiretroviral prophylaxis have resulted in the prevention of HIV in some 670000 children in SSA between 2009 and 2012. ${ }^{14}$ However, the lack of involvement of male partners in such PMTCT services has been found to discourage pregnant women from taking part in the services, and hence may result in even more new paediatric infections. ${ }^{5}{ }^{6}$ Consequently, there has been increasing need to develop approaches 
that can get male partners involved in PMTCT services since their involvement will be crucial in achieving a sustained reduction of mother-to-child transmission of HIV, which is an important step towards the attainment of the elimination of HIV transmission from mother-to-child by $2030 .^{7}$

Several approaches that have been used to improve on the male involvement in PMTCT services involve randomised controlled trials (RCTs) and cohort study designs, but evidence from a systematic review is limited. Sherr and Croome ${ }^{8}$ attempted a review that was aimed at exploring these approaches, but the findings from this review were limited to communication between couples and community involvement approaches. Limitations of this review include: database search limited to Medline, PsycINFO and Cochrane database; article selection by a single author and no flow chart showing how articles were screened; no assessment of the quality of articles to be included; no conference abstract used; no experts consulted; and articles limited to English language. All these caveats strongly suggest the review was not sufficiently systematic, and the high risk of selection bias suggests that not all the studies at the time were included. In addition, the more recent approaches that were attempted and were not included in their review comprise community-based interventions $;{ }^{9}$ home visits; ${ }^{10}$ selftesting; ${ }^{11}$ use of peer-based and structural strategies; ${ }^{12}$ communication among spouses ${ }^{13}$ HIV status disclosure ${ }^{14}$ and co-enrolment of partners. ${ }^{15}$

The evidence on the impact of male partner involvement approaches on PMTCT services has been contrasting. A systematic review by Brusamento $e t a l^{16}$ explored the impact on some services which included: HIV counselling and testing; antiretroviral prophylaxis in women and infants; and enrolment into care. This systematic review was rigorous in methodology as it explored several databases and thoroughly assessed the quality of studies. However, the investigators limited the search to articles published after the year 2000 despite evidence that mother-to-child transmission of HIV prevention strategies were reported as early as 1985 by the Centres for Disease Control and Prevention (CDC). ${ }^{17}$ Furthermore, only one study at the time of completion of the literature search met the inclusion criteria, and hence only information from that single study was used in providing evidence on the impact of male participation on PMTCT services. The evidence from this single included study suggested that male partner involvement has a negative impact on the delivery of PMTCT services. This finding is not consistent with many currently published studies that have reported a positive impact of male partner involvement on some primary PMTCT services. For example, Weiss $e t a l^{18}$ has reported an increase in infant prophylaxis, Myer et $a l^{15}$ reported an increase in ART initiation rates, ${ }^{19}$ Kalembo et $a l^{20}$ reported impact on the proportion of hospital deliveries, Osoti et $a l^{11}$ reported the impact on HIV status disclosure and Orne-Gliemann et $a l^{21}$ have reported the impact on antenatal HIV testing in pregnant women. Other recent studies have reported the impact on secondary PMTCT services such as condom use among partners,${ }^{20}$ uptake of identification of serodiscordant couples, ${ }^{11}$ reduction of intimate partner violence,$^{22}$ enrolment and retention in care. ${ }^{9}$ Therefore, a systematic review to address this contrasting evidence is needed.

The gaps presented in the studies above prompted the need to conduct an up-to-date systematic review on the approaches that have been used in improving on male partner involvement, and the impact these have had on PMTCT services such as counselling and testing by couples, hospital deliveries, HIV disclosure and antiretroviral initiation.

\section{General objective}

The overall aim of the study will be to determine the approaches used to improve male participation in PMTCT of HIV, and the impact that these approaches have on the PMTCT of HIV services in SSA.

\section{Research questions}

What approaches have been used to improve on male involvement in PMTCT in SSA?

What impacts does male involvement have on the uptake of PMTCT services such as couple testing and counselling, and couple and infant initiation on ART in SSA?

\section{Specific research objectives}

1. To develop a literature search strategy for the approaches used in improving male partner participation in PMTCT of HIV and assess the impact these approaches have on the uptake of PMTCT of HIV services in SSA.

2. To screen all the identified studies in (1) for relevance to the research questions.

3. To critically appraise the literature obtained from objective (2).

4. To extract relevant data from studies in (3) on approaches used in improving male partner participation in PMTCT services in SSA.

5. To extract relevant data from studies in (3) on the impact of the approaches in (4) on PMTCT of HIV services in SSA.

6. To draw conclusions on the impacts of approaches used in improving male partner involvement on the delivery of PMTCT services such as couples counselling and testing, hospital deliveries, HIV disclosure, and antiretroviral initiation by couples.

\section{METHODS}

\section{Eligibility criteria}

Study designs

The review will include RCTs, cluster randomised trials, non-randomised controlled studies, cohort studies (both prospective and retrospective), and repeated cross- 
sectional studies. One time cross-sectional studies and case-control studies will be excluded because these will not present any evidence of the impact of male partner involvement. The studies that will be included to answer the research question should investigate an approach or approaches used in improving male partner involvement in PMTCT, and provide data on the impact of the approach(es) on the delivery of PMTCT services at any level.

\section{Participants}

Studies that explore the approaches towards involving male partners irrespective of age who have pregnant partners irrespective of marital status, living in the same house or not, will be included. No distinction will be made with respect to maternal age or number of pregnancies. Interventions directed to all males and/or pregnant partners and infants will be included.

\section{Study setting}

Since our review is focused on the SSA region, only studies carried out in this setting will be included. Community-based studies, clinic-based or home-based studies will all be included. Studies carried out in urban and rural settings will be included. Studies will be included irrespective of language, year of publication and duration of follow-up.

\section{Source of information}

The methods of this systematic review will be reported in accordance with the Preferred Reporting Items for Systematic Reviews and Meta-Analysis Protocols (PRISMA-P) $2015 .^{23}$ The items of these checklists as fulfilled by this protocol are shown in online supplementary table S1.

\section{Search strategy and study identification \\ Main database search}

Using medical subject headings $(\mathrm{MeSH})$ and text key words, literature will be searched relating to male partner participation and PMTCT in SSA. The databases to be searched include OVID Medline, OVID Embase, PubMed, PsycINFO, Cochrane library, Cochrane database of controlled trials, and ClinicalTrials.gov, WHO database of controlled trials.

A detailed planned search strategy for MEDLINE and EMBASE is shown in box 1 .

\section{Reference list}

The reference list of relevant articles will be searched to identify any relevant studies.

After identifying the main relevant studies, the journal in which these were published will be handsearched for any unidentified studies. These journals will be listed in the final review.

For studies that will be selected for critical appraisal, the authors will be contacted by email for the existence of any unpublished literature or research in progress or unpublished data.

\section{Grey literature}

Other general and less precise search engines such as Google and Google scholar will be searched using key words like 'male partner' and 'HIV', 'male partner' and 'PMTCT', and 'male'. This search will help obtain grey literature that will be screened by the two reviewers.

\section{Study records}

\section{Data management}

All identified studies shall be uploaded into the Mendeley software V.1.15.3 for de-duplication of records. The full texts and abstracts will be uploaded into the Rayyan software to facilitate internet-based collaboration between investigators during the selection of articles. A standard pretested questionnaire containing inclusion and exclusion criteria will be drafted to guide the screening of titles and abstracts.

\section{Screening}

Various folders will be created in the Mendeley reference management software, and duplicate results will be removed using the reference management system. Two independent reviewers (the principal investigator and the study supervisor) will screen titles of search results and abstracts. Reviewers will settle any disagreements using the inclusion criteria and outcomes of interest, and a third reviewer will be sought in case of any difficulty to arrive at an agreement by the other two reviewers. Excluded studies will be filed in different folders with the reasons for exclusion clearly stated. A PRISMA flow diagram will reflect this process.

\section{Data extraction}

A standardised data extraction sheet will be produced in Excel V.2013, and data from the different studies will be extracted by two independent reviewers. These sheet will be pretested and amended by two investigators (NFT and CJ) before data collection begins. Should any disagreement between reviewers arise, it will be settled by discussion with a third reviewer (ITRK).

\section{Data items}

The data extracted will include the following items:

- Authorship, year of study, and journal

- Country where study was conducted

- Objectives

- Study design

- Inclusion and exclusion criteria

- Study duration

- Sample size

- Characteristics of participants

- Services targeted by approaches

- Study setting (clinic based, community based or home based) 
Box 1 Detailed planned search strategy

SEARCH STRATEGY FOR MEDLINE, EMBASE AND HPSI

Database: Ovid MEDLINE(R) <1946 to February Week 2 2016>, Embase <1996 to 2016 Week 08>, Health and Psychosocial Instruments $<1985$ to January 2016>

Search Strategy:

1. ${ }^{*} \mathrm{HIV} /$

2. *HIV infection/

3. human immunodeficiency virus.mp. [mp=ti, ab, ot, nm, hw, kf, px, rx, ui, an, tn, dm, mf, dv, kw, ac, sh, de, md, ip, vo, pg, sd, jn, pb, $\mathrm{yr}$, ar, bs, bt, cf, dp, ja, pa, so]

4. human immuno-deficiency virus.mp. [mp=ti, ab, ot, nm, hw, kf, px, rx, ui, an, tn, dm, mf, dv, kw, ac, sh, de, md, ip, vo, pg, sd, jn, pb, $\mathrm{yr}, \mathrm{ar}, \mathrm{bs}, \mathrm{bt}, \mathrm{cf}, \mathrm{dp}, \mathrm{ja}, \mathrm{pa}, \mathrm{so}]$

5. human immune-deficiency virus.mp. [mp=ti, ab, ot, nm, hw, kf, px, rx, ui, an, tn, dm, mf, dv, kw, ac, sh, de, md, ip, vo, pg, sd, jn, pb, $\mathrm{yr}, \mathrm{ar}, \mathrm{bs}, \mathrm{bt}, \mathrm{cf}, \mathrm{dp}, \mathrm{ja}, \mathrm{pa}, \mathrm{so}]$

6. human immunedeficiency virus.mp. [mp=ti, ab, ot, nm, hw, kf, px, rx, ui, an, tn, dm, mf, dv, kw, ac, sh, de, md, ip, vo, pg, sd, jn, pb, $\mathrm{yr}, \mathrm{ar}, \mathrm{bs}, \mathrm{bt}, \mathrm{cf}, \mathrm{dp}$, ja, pa, so]

7. (human immun* and deficiency virus).mp. [mp=ti, ab, ot, nm, hw, kf, px, rx, ui, an, tn, dm, mf, dv, kw, ac, sh, de, md, ip, vo, pg, sd, jn, pb, yr, ar, bs, bt, cf, dp, ja, pa, so]

8. ${ }^{*} \mathrm{AIDS} / \mathrm{pC}$

9. acquired immune-deficiency syndrome.mp. [mp=ti, ab, ot, nm, hw, kf, px, rx, ui, an, tn, dm, mf, dv, kw, ac, sh, de, md, ip, vo, pg, sd, jn, pb, yr, ar, bs, bt, cf, dp, ja, pa, so]

10. acquired immunedeficiency syndrome.mp. [mp=ti, ab, ot, nm, hw, kf, px, rx, ui, an, tn, dm, mf, dv, kw, ac, sh, de, md, ip, vo, pg, sd, jn, $\mathrm{pb}, \mathrm{yr}, \mathrm{ar}, \mathrm{bs}, \mathrm{bt}, \mathrm{cf}, \mathrm{dp}, \mathrm{ja}, \mathrm{pa}, \mathrm{so}]$

11. acquired immunodeficiency syndrome.mp. [mp=ti, ab, ot, nm, hw, kf, px, rx, ui, an, tn, dm, mf, dv, kw, ac, sh, de, md, ip, vo, pg, sd, jn, pb, yr, ar, bs, bt, cf, dp, ja, pa, so]

12. (acquired immune* and deficiency syndrome).mp. [mp=ti, ab, ot, nm, hw, kf, px, rx, ui, an, tn, dm, mf, dv, kw, ac, sh, de, md, ip, vo, $\mathrm{pg}, \mathrm{sd}, \mathrm{jn}, \mathrm{pb}, \mathrm{yr}, \mathrm{ar}, \mathrm{bs}, \mathrm{bt}, \mathrm{cf}, \mathrm{dp}, \mathrm{ja}, \mathrm{pa}, \mathrm{so}]$

13. Infectious disease transmission.mp. [mp=ti, ab, ot, nm, hw, kf, px, rx, ui, an, tn, dm, mf, dv, kw, ac, sh, de, md, ip, vo, pg, sd, jn, pb, $\mathrm{yr}, \mathrm{ar}, \mathrm{bs}, \mathrm{bt}, \mathrm{cf}, \mathrm{dp}, \mathrm{ja}, \mathrm{pa}, \mathrm{so}]$

14. vertical transmission.mp. [mp=ti, ab, ot, nm, hw, kf, px, rx, ui, an, tn, dm, mf, dv, kw, ac, sh, de, md, ip, vo, pg, sd, jn, pb, yr, ar, bs, bt, cf, dp, ja, pa, so]

15. vertical infectious disease transmission.mp. [mp=ti, ab, ot, nm, hw, kf, px, rx, ui, an, tn, dm, mf, dv, kw, ac, sh, de, md, ip, vo, pg, sd, jn, pb, yr, ar, bs, bt, cf, dp, ja, pa, so]

16. * ${ }^{*}$ Sexually Transmitted Diseases/pc

17. * ${ }^{*}$ Sexually Transmitted Diseases, viral/

18. mother-to-child transmission.mp. [mp=ti, ab, ot, nm, hw, kf, px, rx, ui, an, tn, dm, mf, dv, kw, ac, sh, de, md, ip, vo, pg, sd, jn, pb, yr, ar, bs, bt, cf, dp, ja, pa, so]

19. Parent-to-child transmission.mp. [mp=ti, ab, ot, nm, hw, kf, px, rx, ui, an, tn, dm, mf, dv, kw, ac, sh, de, md, ip, vo, pg, sd, jn, pb, yr, ar, bs, bt, cf, dp, ja, pa, so]

20. Maternal-to-child transmission.mp. [mp=ti, ab, ot, nm, hw, kf, px, rx, ui, an, tn, dm, mf, dv, kw, ac, sh, de, md, ip, vo, pg, sd, jn, pb, yr, ar, bs, bt, cf, dp, ja, pa, so]

21. maternal-fetal infection transmission.mp. [mp=ti, ab, ot, nm, hw, kf, px, rx, ui, an, tn, dm, mf, dv, kw, ac, sh, de, md, ip, vo, pg, sd, jn, $\mathrm{pb}, \mathrm{yr}, \mathrm{ar}, \mathrm{bs}, \mathrm{bt}, \mathrm{cf}, \mathrm{dp}, \mathrm{ja}, \mathrm{pa}, \mathrm{so}]$

22. MTCT.mp. [mp=ti, ab, ot, nm, hw, kf, px, rx, ui, an, tn, dm, mf, dv, kw, ac, sh, de, md, ip, vo, pg, sd, jn, pb, yr, ar, bs, bt, cf, dp, ja, pa, So]

23. PMTCT.mp. [mp=ti, ab, ot, nm, hw, kf, px, rx, ui, an, tn, dm, mf, dv, kw, ac, sh, de, md, ip, vo, pg, sd, jn, pb, yr, ar, bs, bt, cf, dp, ja, pa, so]

24. pPTCT.mp. [mp=ti, ab, ot, nm, hw, kf, px, rx, ui, an, tn, dm, mf, dv, kw, ac, sh, de, md, ip, vo, pg, sd, jn, pb, yr, ar, bs, bt, cf, dp, ja, $\mathrm{pa}, \mathrm{so}]$

25. male partner*.mp. [mp=ti, ab, ot, nm, hw, kf, px, rx, ui, an, tn, dm, mf, dv, kw, ac, sh, de, md, ip, vo, pg, sd, jn, pb, yr, ar, bs, bt, cf, $\mathrm{dp}, \mathrm{ja}, \mathrm{pa}, \mathrm{so}]$

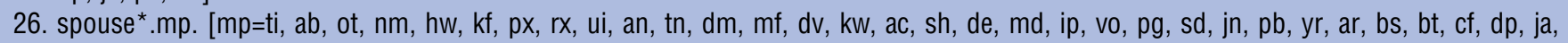
pa, so]

27. husband*.mp. [mp=ti, ab, ot, nm, hw, kf, px, rx, ui, an, tn, dm, mf, dv, kw, ac, sh, de, md, ip, vo, pg, sd, jn, pb, yr, ar, bs, bt, cf, dp, ja, $\mathrm{pa}, \mathrm{so}]$

28. couple*.mp. [mp=ti, ab, ot, nm, hw, kf, px, rx, ui, an, tn, dm, mf, dv, kw, ac, sh, de, md, ip, vo, pg, sd, jn, pb, yr, ar, bs, bt, cf, dp, ja, $\mathrm{pa}, \mathrm{so}]$

29. fathers*.mp. [mp=ti, ab, ot, nm, hw, kf, px, rx, ui, an, tn, dm, mf, dv, kw, ac, sh, de, md, ip, vo, pg, sd, jn, pb, yr, ar, bs, bt, cf, dp, ja, $\mathrm{pa}, \mathrm{so}]$

30. men*.mp. [mp=ti, ab, ot, nm, hw, kf, px, rx, ui, an, tn, dm, mf, dv, kw, ac, sh, de, md, ip, vo, pg, sd, jn, pb, yr, ar, bs, bt, cf, dp, ja, pa, so] 


\section{Box 1 Continued}

31. sexual partner*.mp. [mp=ti, ab, ot, nm, hw, kf, px, rx, ui, an, tn, dm, mf, dv, kw, ac, sh, de, md, ip, vo, pg, sd, jn, pb, yr, ar, bs, bt, cf, $\mathrm{dp}, \mathrm{ja}, \mathrm{pa}, \mathrm{so}]$

32. prevention *.mp. [mp=ti, ab, ot, nm, hw, kf, px, rx, ui, an, tn, dm, mf, dv, kw, ac, sh, de, md, ip, vo, pg, sd, jn, pb, yr, ar, bs, bt, cf, dp, ja, pa, so] (924849)

33. reduc*.mp. [mp=ti, ab, ot, nm, hw, kf, px, rx, ui, an, tn, dm, mf, dv, kw, ac, sh, de, md, ip, vo, pg, sd, jn, pb, yr, ar, bs, bt, cf, dp, ja, $\mathrm{pa}, \mathrm{so}]$

34. educat*.mp. [mp=ti, ab, ot, nm, hw, kf, px, rx, ui, an, tn, dm, mf, dv, kw, ac, sh, de, md, ip, vo, pg, sd, jn, pb, yr, ar, bs, bt, cf, dp, ja pa, so]

35. (awareness or health promotion or safe sex or condom* or efficacy or efficiency or behav* or test* ${ }^{\star}$ or notif* or contact tracing ${ }^{\star}$ or prophylaxis*).mp. [mp=ti, ab, ot, nm, hw, kf, px, rx, ui, an, tn, dm, mf, dv, kw, ac, sh, de, md, ip, vo, pg, sd, jn, pb, yr, ar, bs, bt, cf, $\mathrm{dp}, \mathrm{ja}, \mathrm{pa}, \mathrm{so}]$

36. 1 or 2 or 3 or 4 or 5 or 6 or 7 or 8 or 9 or 10 or 11 or 12

37. 13 or 14 or 15 or 16 or 17 or 18 or 19 or 20 or 21 or 22 or 23 or 24

38. 25 or 26 or 27 or 28 or 29 or 30 or 31

39. 32 or 33 or 34 or 35

40. 36 and 37 and 38 and 39 .

- Type of approaches used (such as psychosocial, medical, community and peer group)

- Expected outcomes

- Results of actual outcomes measured, that is the effect sizes: mean differences, differences in proportions, relative risks (RRs), ORs, p values.

\section{Outcomes of interest}

The primary outcomes will include:

- Proportion of pregnant women attending antenatal clinics

- Proportion of couples counselled and tested for HIV

- Proportion of women testing for HIV

- Proportion of male partners testing for HIV

- Proportion of pregnant women and infants who are receiving antiretrovirals (ARVs)

The secondary outcomes will include:

- Proportion of couples who use condoms

- Proportion of mothers who engage in safe infant breastfeeding practices

- Proportion of mothers who come for hospital deliveries

- Proportion of women who use/intend to use family planning.

\section{Assessment of methodological quality and risk of bias Critical appraisal}

Critical appraisal will be done to assess for bias and confounding. Using the Critical Appraisal Skills Programme (CASP) tool for observational and experimental studies, a template for results of critical appraisal will be developed to capture the following aspects:

- Aims of the study and if these were stated clearly

- Whether the right study design was used

- Method of recruitment of participants

- Exclusion criteria

- Response and follow-up rate

- Extent to which the sample is representative

- Presentation of results

- Consistency with other studies.
The critical appraisal will be carried out independently by two investigators (NFT and CJ). Strengths and weaknesses of the methodology of each study will be considered individually in order to arrive at a decision.

\section{Assessment of risk of bias}

This assessment will be carried out independently by the two reviewers (NFT and CJ). Further assessment of risk of bias and quality of the studies will be done using the Jadad scale ${ }^{24}$ for randomised trials, and the STROBE checklist $^{25}$ for observational studies. Box 2 shows the items in the Jadad scale.

Box 2 The Jadad scale for assessment of quality of randomised trials

Quality assessment using the Jadad scale for randomised controlled trials

A commonly used three-item, five-point quality scale was used to rate the quality of the trials (Jadad et $\left.a R^{4}\right)$. The minimum score possible for inclusion of a study in the review was 2 (1 point each for randomisation and double-blinding). The maximum score possible was 5 (2 points for descriptions of randomisation, 2 points for descriptions of double blinding, and 1 point for descriptions of withdrawals).

How points are awarded:

1. Is the study randomised? If yes, +1 point.

2. Is the randomisation procedure appropriate and reported in the study?

3. If yes, +1 point. If no, delete all points awarded for randomisation.

4. Is the study double-blinded? If yes, +1 point.

5. Is the double-blinding method appropriate and reported in the study?

6. If yes, +1 point. If no, delete all points awarded for double-blinding.

7. Are the reasons for patient withdrawals and dropouts described for each treatment group?

8. If yes, +1 point. 
Data synthesis, analysis and assessment for heterogeneity

The information on the data extraction sheet will be exported to STATA V.12. Heterogeneity will be tested in STATA V.12 using the $Q$ statistic and $\mathrm{I}^{2}$, with level of statistical significance set at 0.05 . If the $\mathrm{I}^{2}=25-49 \%$ we will consider a 'low' heterogeneity, $\mathrm{I}^{2}=50-74 \%$ will be considered as 'moderate' heterogeneity and $\mathrm{I}^{2} \geq 75 \%$ will be considered as 'high' heterogeneity. If data are inappropriate for quantitative synthesis, we will proceed with qualitative synthesis only. With low heterogeneity, we will proceed with meta-analysis with pooled effect sizes presented as forest plots. The random effect model will be used to produce forest plots. The effect sizes could be mean differences, differences in proportions, ORs, RRs, and $\mathrm{p}$ values. Publication bias will be assessed using funnel plots and the Egger's test.

\section{Sensitivity analysis}

If substantial heterogeneity is detected, subgroup analysis will be performed to identify possible sources of heterogeneity with the following grouping variables: age group, study setting, study quality, study design, method of randomisation. Subgroup differences will be described and interpreted accordingly.

\section{Confidence in cumulative evidence}

The strength of evidence provided by the studies included in this review was obtained by using the Grading of Recommendations Assessment Development and Evaluation (GRADE) approach. This approach will assess the risk of bias, consistency and publication bias. Studies will be described as 'high', 'moderate', 'low' and 'very low' quality if further research is unlikely to change the estimates, likely to have considerable impact on effect estimates, capable of changing effect sizes, respectively.

\section{Reporting of this review}

This review will be published according to PRISMA guidelines and the PRISMA checklist will be published with the completed report. ${ }^{23}$

\section{Potential amendments}

No further protocol amendment is envisaged in order to avoid any possibility of reporting bias. However, any amendment in the final report will be explained clearly.

\section{Ethical consideration and dissemination of findings}

This study will require no ethical approval since no intervention will be carried out on a human subject, and the studies that will be selected for the review had already obtained ethical approval before they were conducted. The studies will be published studies, conference abstracts, and grey literature that are 'freely' available and accessible.

It is expected that this study will provide evidence on the impact of approaches that have been used so far in improving the involvement of male partners in PMTCT. We expect to present approaches that have improved the proportion of couples testing for HIV, improved the rate of HIV status disclosure among couples, and improved the proportion of couples and infants on ART in SSA. This evidence is currently needed by Ministries of Health, HIV funding agencies, and HIV implementation partners to guide the efforts towards effectively involving male partners in PMTCT to ensure the elimination of mother-to-child transmission of HIV in sub-Saharan Africa. ${ }^{16}$

\section{Author affiliations}

${ }^{1}$ Ministry of Public Health, Cameroon

${ }^{2}$ Institute of Health and Well-being, University of Glasgow, Glasgow, UK

${ }^{3}$ Public Health Protection Unit, NHS Greater Glasgow and Clyde, UK

Acknowledgements The authors would like to thank the University of Glasgow Library for assess to OVID Medline, OVID Embase, and other search databases.

Contributors NFT and CJ conceived the paper. NFT made the first draft. NFT, ITRK and CJ reviewed several versions of the manuscript. All the authors read and approved the final manuscript.

Competing interests None declared.

Provenance and peer review Not commissioned; externally peer reviewed.

Open Access This is an Open Access article distributed in accordance with the Creative Commons Attribution Non Commercial (CC BY-NC 4.0) license, which permits others to distribute, remix, adapt, build upon this work noncommercially, and license their derivative works on different terms, provided the original work is properly cited and the use is non-commercial. See: http:// creativecommons.org/licenses/by-nc/4.0/

\section{REFERENCES}

1. UNAIDS. The Gap Report. The beginning of the end of the AIDS epidemic. September 2014. ISSN 978-92-9253-062-4.

2. UNAIDS. The Gap Report. UNAIDS report on Global AIDS epidemic 2013. ISBN 978-92-9253-032-7

3. UNAIDS. Prevention of HIV transmission from mother to child. Strategic options. 1999. 99.40E

4. UNAIDS. Together we will end AIDS. UNAIDS, 2012. ISSN 978-92-9173-974-5.

5. Bajunirwe F, Muzoora M. Barriers to the implementation of programs for the prevention of mother-to-child transmission of HIV: a cross-sectional survey in rural and urban Uganda. AIDS Res Ther 2005;2:10

6. Sarker M, Sanou A, Snow R, et al. Determinants of HIV counselling and testing participation in a prevention of mother-to-child transmission programme in rural Burkina Faso. Trop Med Int Health 2007;12:1475-83.

7. UNAIDS. Fast-track: ending the AIDS epidemic by 2030. UNAIDS, 2014. ISSN 978-92-9253-063-1.

8. Sherr L, Croome N. Involving fathers in prevention of mother to child transmission initiatives-what the evidence suggests. J Int AIDS Soc 2012;15(Suppl 2):17378.

9. Rosenberg NE, van Lettow M, Tweya $\mathrm{H}$, et al. Improving PMTCT uptake and retention services through novel approaches in peer-based family-supported care in the clinic and community: a 3-arm cluster randomized trial (PURE Malawi). J Acquir Immune Defic Syndr 2014;67(Suppl 2):S114-19.

10. Osoti AO, John-Stewart G, Kiarie JN, et al. Home-based HIV testing for men preferred over clinic-based testing by pregnant women and their male partners, a nested cross-sectional study. BMC Infect Dis 2015;15:298.

11. Osoti AO, John-Stewart G, Kiarie J, et al. Home visits during pregnancy enhance male partner HIV counselling and testing in Kenya: a randomized clinical trial. AIDS 2014;28:95-103.

12. Mphonda SM, Rosenberg NE, Kamanga E, et al. Assessment of peer-based and structural strategies for increasing male participation in an antenatal setting in Lilongwe, Malawi. Afr J Reprod Health 2014;18:97-104.

13. Desgrées-Du-Loû A, Brou H, Djohan G, et al. Beneficial effects of offering prenatal HIV counselling and testing on developing a HIV 
preventive attitude among couples. Abidjan, 2002-2005. AIDS Behav 2009;13:348-55.

14. Jasseron C, Mandelbrot L, Dollfus C, et al. Non-disclosure of a pregnant woman's HIV status to her partner is associated with non-optimal prevention of mother-to-child transmission. AIDS Behav 2013;17:488-97.

15. Myer L, Abrams EJ, Zhang Y, et al. Family matters: co-enrollment of family members into care is associated with improved outcomes for HIV-infected women initiating antiretroviral therapy. J Acquir Immune Defic Syndr 2014;67(Suppl 4):S243-9.

16. Brusamento $\mathrm{S}$, Ghanotakis E, Tudor Car L, et al. Male involvement for increasing the effectiveness of prevention of mother-to-child HIV transmission (PMTCT) programmes. Cochrane Database Syst Rev 2012;10:CD009468.

17. CDC. Recommendations for assisting in the prevention of perinatal transmission of human T-lymphotropic virus type III/ lymphadenopathy-associated virus and acquired immunodeficiency syndrome. MMWR Morb Mortal Wkly Rep 1985;34:741-32.

18. Weiss SM, Peltzer K, Villar-Loubet O, et al. Improving PMTCT uptake in rural South Africa. J Int Assoc Provid AIDS Care 2014;13:269-76.

19. Herce ME, Mtande T, Chimbwandira F, et al. Supporting option B+ scale up and strengthening the prevention of mother-to-child transmission cascade in central Malawi: results from a serial cross-sectional study. BMC Infect Dis 2015;15:328.

20. Kalembo FW, Zgambo M, Mulaga AN, et al. Association between male partner involvement and the uptake of prevention of mother-to-child transmission of HIV (PMTCT) interventions in Mwanza district, Malawi: a retrospective cohort study. PLOS ONE 2013;8:e66517.

21. Orne-Gliemann J, Balestre E, Tchendjou P, et al. Increasing HIV testing among male partners. AIDS 2013;27:1167-77.

22. Rosenberg NE, Mtande TK, Saidi F, et al. Recruiting male partners for couple HIV testing and counselling in Malawi's option B+ programme: an unblinded randomised controlled trial. Lancet HIV 2015;2:e483-91.

23. Moher D, Liberati A, Tetzlaff J, et al. The PRISMA group. Preferred Reporting Items for Systematic Reviews and Meta-Analysis: The PRISMA Statement. PLoS Med 2009;6:e1000097.

24. Jadad AR, Moore RA, Carroll D, et al. Assessing the quality of reports of randomized clinical trials: is blinding necessary? Control Clin Trials 1996;17:1-12.

25. Vandenbroucke JP, von Elm E, Altman DG, et al. Strengthening the Reporting of Observational Studies in Epidemiology (STROBE): explanation and elaboration. Int $J$ Surg 2014;12:1500-24. 